\title{
Intussusception of the Small Intestine Caused by a Primary Melanoma?
}

\author{
M. Schoneveld K. De Vogelaere N. Van De Winkel
}

\section{A. Hoorens G. Delvaux}

UZ Brussel, Brussels, Belgium

\section{Key Words}

Melanoma $\cdot$ Small bowel $\cdot$ Intussusception

\begin{abstract}
Although the gastrointestinal tract is a fairly frequent site of melanoma metastases, reports of small bowel intussusception caused by melanoma are very rare. We report the case of a 77-year-old man who was admitted to our hospital with epigastric pain, melena and anaemia. After clinical examination, laboratory evaluation and radiological work-up the diagnosis of a jejunal intussusception was made. Exploratory laparoscopy revealed a large tumour arising from the jejunum, approximately $20 \mathrm{~cm}$ distal to the angle of Treitz. Small bowel resection with an end-to-end anastomosis was performed. Histological examination showed an intestinal melanoma. There are different theories concerning the origin of malignant melanoma in the small bowel. Although the small and large intestines normally contain no melanocytes, these cells have occasionally been found in the alimentary and respiratory tracts and even in lymph nodes, which supports the theory of a primary origin of melanoma at these sites. Since this was a solitary intestinal lesion and there was no history of cutaneous melanoma, we conclude that this could be an example of a very rare primary melanoma of the small intestine.
\end{abstract}

\section{Introduction}

Most melanomas of the small intestine are metastases from primary cutaneous lesions. They are usually diagnosed when a complication occurs such as occlusion, bleeding, anaemia and perforation of the intestine, but small bowel intussusception is also described [1]. In a review of 84,836 cases of melanoma, $91.2 \%$ were cutaneous, $5.2 \%$ ocular, $2.2 \%$ of unknown primary site and only $1.3 \%$ originated from the gastrointestinal mucosa [2]. The prevalence of malignant melanoma in the gastrointestinal tract with no evidence of a primary lesion in the skin or any other site is extremely low. 


\section{Case Report}

A 77-year-old man was admitted to our hospital with epigastric pain, melena and anaemia. He had a history of a right hemicolectomy for an adenocarcinoma of the colon in 2005 (T2N 0M0) with a disease-free follow-up of almost 5 years. On physical examination, moderate, diffuse tenderness over the epigastrium was noted. Routine laboratory data revealed severe anaemia with a haemoglobin of $5 \mathrm{~g} / \mathrm{dl}$. Carcinoembryonic antigen levels were normal. Neither gastroscopy nor colonoscopy was abnormal. Computed tomography of the abdomen demonstrated characteristic 'target sign' and proximal small bowel dilatation, strongly suggesting jejunal intussusception ( $\underline{\text { fig. } 1 \text { ). }}$.

A diagnostic laparoscopy was done which revealed a large tumour arising from the antimesenteric margin of the jejunum, approximately $20 \mathrm{~cm}$ distal of the angle of Treitz (ig. $2 \underline{\text { fig. } 3}$ ). No macroscopic signs of metastasis were detected in any other organ in the abdominal cavity. Small bowel resection with wide excision of both the lesion and mesentery, followed by an end-to-end anastomosis, was performed. There were no postoperative complications and the patient was discharged on the 5th postoperative day. Macroscopic and microscopic examination, including immunohistochemistry, revealed a malignant melanoma $6 \mathrm{~cm}$ in diameter without local lymph node metastasis (fig. 4). Histologically, a clear cell sarcoma can resemble a melanoma of the gastrointestinal tract with little melanin secretion. However this diagnosis seemed unlikely upon genetic analysis, as there was no translocation in the EWSR1 gene, which is a typical marker for clear cell sarcoma. After the diagnosis of melanoma was established, detailed postoperative examination failed to identify any other primary melanoma lesion [3].

\section{Discussion}

Intussusception is a rare cause of mechanical intestinal obstruction in adults, and diagnosis of intussusception in an adult patient strongly suggests the presence of a malignant pathology [4]. Frequently encountered malignant neoplasms of the small bowel include adenocarcinomas, neuroendocrine tumours, lymphoma, gastrointestinal stromal tumours and metastases [5]. Further, the small intestine is the most common site of melanoma metastases to the gastrointestinal tract. Most melanomas in the small intestine are metastases from primary cutaneous lesions. However, melanoma can also develop as a primary mucosal tumour in the gastrointestinal tract [4].

Whether a patient's intestinal melanoma is a primary lesion or the result of metastatic disease is commonly unanswered, and is thus a controversial subject. Some authors suggest that primary melanoma of the small intestine does not exist as a separate entity and that all small bowel melanomas are metastatic lesions from unknown or regressed primary cutaneous melanoma. In the literature, there are some cases and series which describe primary gastrointestinal melanomas $[6,7]$.

Several theories exist concerning the origin of malignant melanoma in the small bowel. Although the small and large intestines normally contain no melanocytes, these cells have occasionally been found in the alimentary tract, the respiratory tract and even in lymph nodes, which supports the theory of a primary origin of melanoma at these sites. The proposed primary sources from which melanomas are derived are melanoblastic cells of the neural crest. Multipotential cells migrate through the body and can arrive in the bowel via the umbilical-mesenteric canal, where they can differentiate into specialised cells. In the gut, neural crest cells differentiate into amine precursor uptake and decarboxylation cells, which can undergo neoplastic transformation and produce tumours such as gastrinomas, carcinoid tumours or melanomas [8]. Although it is clinically important to distinguish primary from 
metastatic disease, establishing the exact origin of the melanoma lesion can be difficult. Criteria such as those proposed by Sachs et al. [9] can aid in this differentiation. They include the presence of a solitary lesion, the lack of metastatic deposits other than those in the regional lymph nodes, and a disease-free survival after diagnosis of at least 12 months.

Melanomas arising on mucosal surfaces appear to be more aggressive and are associated with a worse prognosis than cutaneous melanomas, with an average 5 -year survival rate of $20 \%$ or less. This may be related to delayed diagnosis and to the rich lymphatic and vascular supply of the mucosa, which may lead to early metastasis [9].

\section{Conclusion}

In conclusion, primary melanoma of the gastrointestinal tract is an extremely rare neoplastic lesion and an unusual cause of intussusception in adults. Regardless of the exact origin of the melanotic lesion, the treatment of choice is extensive surgery, including wide resection of the tumour with wedge resection of the mesentery [4].

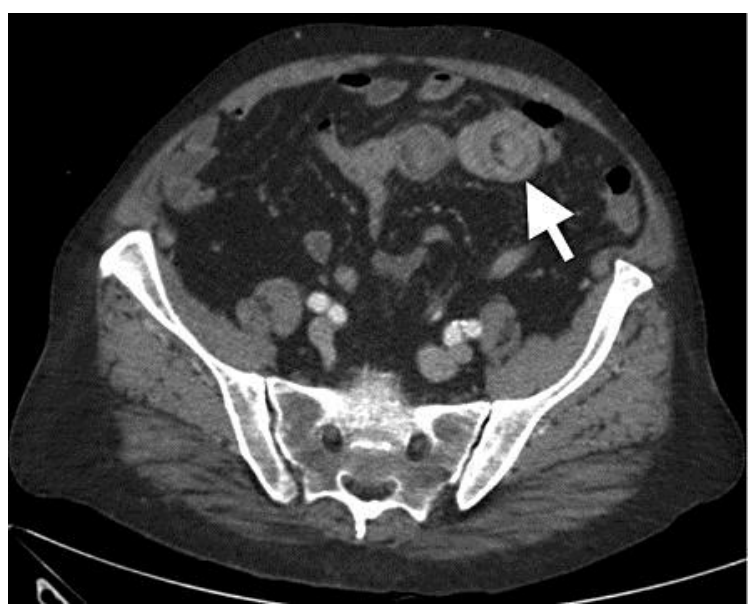

Fig. 1. Computed tomography of the abdomen showed target sign suggesting a jejunal intussusception. 


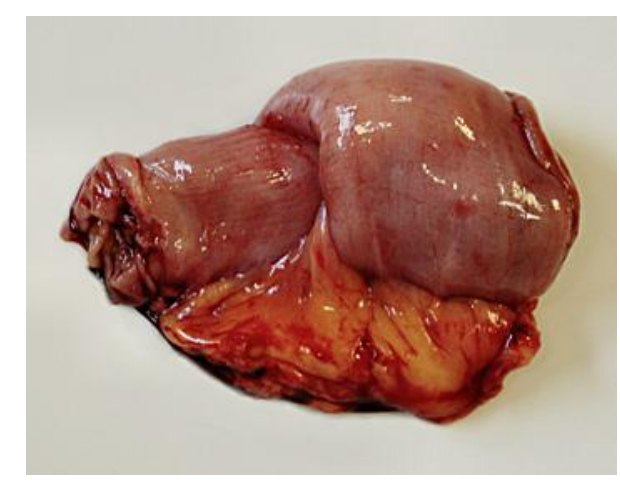

Fig. 2. Surgical specimen. Resected ileal segment showing intussusception.

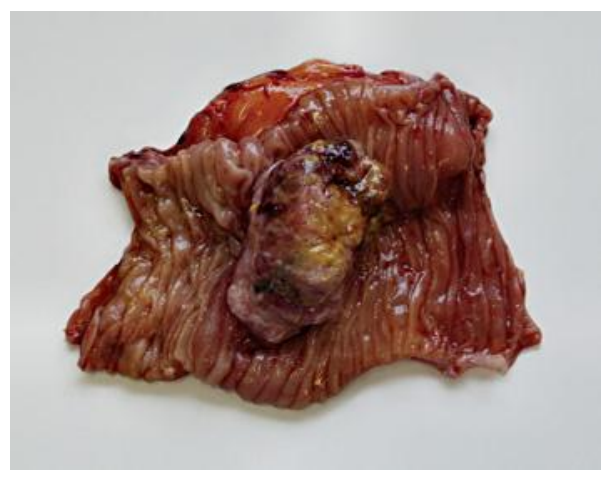

Fig. 3. Surgical specimen. The opened fragment showed a tumoural mass $6 \mathrm{~cm}$ in diameter protruding into the intestinal lumen.

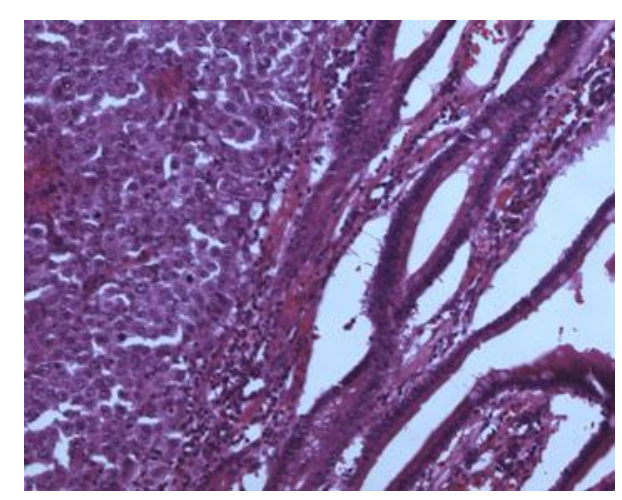

Fig. 4. Microscopic examination showed a dense aggregate of melanoma cells covered by intestinal epithelium $(H \& E, \times 200)$. 


\section{References}

1 Resta G, Anania G, Messina F, et al: Jejuno-jejunal invagination due to intestinal melanoma. World J Gastroenterol 2007;13:310-312.

2 Chang A, Karnell L, Menck H: The National Cancer Data Base report on cutaneous and noncutaneous melanoma: a summary of 84,846 cases from the past decade. Cancer 1998;83:1664-1678.

-3 Song JS, Choi J, Kim J, Jang S, Cho K: Diagnostic utility of EWS break-apart fluorescence in situ hybridization in distinguishing between non-cutaneous melanoma and clear cell sarcoma. Pathol Int 2010;60:608-613.

4 Lens M, Bataille V, Krivokapic Z: Melanoma of the small intestine. Lancet Oncol 2009;10:516-521.

5 Crosby J, Catton C, Davis A, et al: Malignant gastrointestinal stromal tumors of the small intestine: a review of 50 cases from a prospective database. Ann Surg Oncol 2001;8:50-59.

-6 Manouras A, Genetzakis M, Lagoudianakis E, et al: Malignant gastrointestinal melanomas of unknown origin: should it be considered primary? World J Gastroenterol 2007;13:4027-4029.

$\checkmark 7$ Katsourakis A, Noussios G, Alatsakis M, Chatzis I, Chatzitheoklitos E: Primary malignant melanoma of the small intestine: a case report. Acta Chir Belg 2009;109:405-407.

-8 Krausz M, Ariel I, Behar A: Primary malignant melanoma of the small intestine and the APUD cell concept. J Surg Oncol 1978;10:283-288.

9 Sachs D, Lowe L, Chang A, Carson E, Johnson T: Do primary intestinal melanomas exist? J Am Acad Dermatol 1999;41:1042-1044.

Written informed consent was obtained from the patient for publication of this case report and accompanying images. 